\title{
L'impact de l'Enseignement Supérieur sur la Croissance Economique : Une analyse sur données de panel pour un groupe de pays d'Afrique
}

\section{The Impact of Higher Education on Economic Growth: A Panel Data Analysis for a Group of African Countries}

\author{
Oussama ELKHALFI ${ }^{1}$, Rachid CHAABITA ${ }^{2}$, and Chafik GUEMIMI ${ }^{3}$ \\ ${ }^{1}$ Doctorant, Laboratoire de Recherche Actuariat Criminalité Financière et Migration Internationale, Faculté des Sciences Juridique \\ Economiques et Sociales Ain Chock, Casablanca \\ ${ }^{2}$ Enseignant chercheur, Laboratoire de Recherche Actuariat Criminalité Financière et Migration Internationale, Faculté des \\ Sciences Juridique Economiques et Sociales Ain Chock, Casablanca, Université Hassan II de Casablanca-Maroc \\ ${ }^{3}$ Enseignant chercheur, Laboratoire de Recherche Actuariat Criminalité Financière et Migration Internationale, ENSAM \\ Casablanca, Université Hassan II de Casablanca-Maroc
}

\begin{abstract}
Résumé : La relation entre le capital humain et la croissance économique a évoquée une littérature empirique abondante à partir le début des années 90 avec des aboutissements opposés. En fait, la plupart des apports des analyses théoriques ont affirmé que le capital humain joue un rôle positif et significatif sur la croissance économique. Pour notre part, nous envisageons à évaluer cette relation dans l'optique de l'enseignement supérieur et sa qualité dans la croissance et le développement cette fois. À cet égard, nous tendons d'affranchir en séries chronologiques la relation entre l'enseignement supérieur et la croissance économique pour un groupe de pays d'Afrique qui englobe (Maroc, Tunisie, Sénégal et Niger), couvrant la période 2002-2018.Pour ce faire, nous avons opté pour un modèle économétrique panel de type à effets individuels fixes qu'est plus adapté à la structure de nos données. Les résultats auxquels nous avons abouti attestent que plus le stock de capital humain composé par des individus arriveraient à l'université est important, plus le niveau du Pib par tète est élevé et que ce dernier est impacté selon le niveau de la qualité des systèmes d'enseignement supérieur et l'économie des pays.
\end{abstract}

Mots-clés : capital humain, croissance économique, panel, PIB par tête.

\begin{abstract}
The relationship between human capital and economic growth has evoked an abundant empirical literature from the early 1990s with opposing results. In fact, most of the contributions of theoretical analyzes have affirmed that human capital plays a positive and significant role in economic growth. For our part, we plan to assess this relationship from a higher education perspective and its quality in growth and development this time around. In this regard, we tend to break down in time series the relationship between higher education and economic growth for a group of African countries which includes (Morocco, Tunisia, Senegal and Niger), covering the period 2002-2018. To do this, we opted for a panel econometric model of the type with individual fixed effects that is more suited to the structure of our data. The results we have obtained attest that the greater the stock of human capital composed by individuals arriving at university, the higher the level of GDP per head and that the latter is impacted according to the level of quality of the systems of education higher education and the economy of countries.
\end{abstract}

Keywords: human capital, economic growth, panel, GDP per capita. 


\section{INTRODUCTION}

Cet article dans le cadre d'évaluation de l'effet de la qualité de l'enseignement supérieur et ses retombées sur le développement d'une nation, mais cette fois porte sur une vision plus vaste, notamment par le biais de la relation «capital humain et croissance économique » en particulier « enseignent supérieur et croissance. Toutefois, l'interrogation de l'effet du capital humain a eu un intérêt distinct dans les divers travaux théoriques et empiriques traitant l'étude de la croissance économique. L'apparition des naissantes théories de la croissance endogène qui attribuent à l'éducation et à l'aptitude en général une implication fondamentale dans la croissance économique a admis de reproduire les études sur les déterminantes de la croissance. En conséquence, comme les travaux théoriques et empiriques de (Mankiw, Weil et Romer,1992), (Barro, 1991) et (Fleisher et Al,2010) qu'ont arboré l'existence d'un lien cohérent entre l'éducation et la croissance économique ont montré l'existence d'une relation robuste entre l'éducation et la croissance économique par le biais d'une population composée de hautes compétences capable d'inventer, de suivre le progrès techniques et de procurer des nouvelles technologies à travers la formation d'une main d'œuvre qualifiée capable d'innover et d'acquérir des nouvelles technologies qui contribuent d'améliorer et d'augmenter la productivité nationale et sa qualité. Instantanément, il se voit que le niveau de l'éducation acquis par une population reflète une économie forme un facteur indéniable de son succès sur l'échelle de l'économie mondial et aussi sur le niveau de sa propre population.

Certes pays en développement, à l'instar des pays dans notre échantillon, ont opté des nouvelles réformes dans leurs systèmes éducatifs. Ces dernières pointent deux principaux buts. D'un part, répondre aux demandeurs de formation afin de satisfaire les besoins sociaux, et d'autre part, produire une population qualifiée compose de hautes aptitudes et de compétences capables à répondre aux besoins économiques à court et long terme.

À partir de ce constat, et pour bien cerner les divers aspects de la problématique de l'attribution du capital humain en particulier (l'enseignement supérieur) à la croissance économique, nous avons approuvé essentiel de s'interroger sur les escomptes du système éducatif de l'enseignement supérieur sur la croissance d'un panel de pays d'Afrique, en tenant en compte la qualité des systèmes éducatifs. En effet, les indicateurs de qualité de l'éducation, vont contribuer à retrouver le rôle positif du capital humain. Ce qui va donner un plus pour notre propre recherche.

Tout en tenant compte aussi sur la qualité du système éducatif, particulièrement du niveau supérieur et son effet sur la relation éducation-croissance notre problématique sera la suivante :

Quel est l'effet de l'enseignement supérieur sur la croissance économique essentiellement dans les pays à revenu intermédiaire ? Est-ce que cet effet diffère selon le niveau économique de ces pays ? Afin de dégager les résultats obtenus lors de l'estimation des données de panel. Y a-t-il une relation entre l'aspect qualitatif de l'éducation supérieure et la croissance ? Cet effet qualitatif contribue-t-il plus à l'amélioration de cette dernière? Cela permet d'ôter l'importance du rôle interventionniste de l'État dans le domaine de l'éducation marqué par environnement poussé de plus en plus envers la privatisation.

Notre travail se focalisera sur une base de données provienne de la banque mondiale couvrant la période entre 2002-2018.

Afin de donner des éléments de réponse à telles questions dans ce papier, nous serons menés dans un premier lieu à présenter la revue littérature qui comprend les apports théoriques et empiriques qui traitant les divers mécanismes de transmission dont l'éducation en particulier (l'enseignement supérieur) attribue d'une manière directe ou indirecte à la croissance. En deuxième lieu, nous testerons l'effet de l'éducation en particulier (l'enseignement supérieur) sur la croissance économique dans notre échantillon de pays à revenu intermédiaire à travers une modélisation économétrique de type d'un modèle panel. En dernier lieu, nous discuterons nos 
résultats dont l'objectif principal est d'examiner si ces derniers coïncident avec approbations théoriques, afin de savoir si cet effet de l'éducation sur la croissance existe sur le contexte des pays de notre échantillon ce qui nous permet à clarifier les altercations, les ambigüités et l'impact de la qualité du système éducatif sur la fameuse relation éducation-croissance.

\section{REVUE DE LITTERATURE ET DEVELOPPEMENT DES HYPOTHESES}

Pour mettre en évidence le rôle que joue l'éducation en tant que capital humain dans la croissance économique nous faisons appel tout d'abord aux deux types d'approches du capital humain sont utilisées : l'approche par les flux portant sur les variables de taux contre l'approche de stock concernant les stocks du capital humain, après nous abordons les théories les plus représentatives et celles qui ont le plus travaillé sur les questions d'économie politique d'éducation. Il s'agit de la théorie du capital humain et les théories de la croissance endogène. Enfin, nous faisons le tour sur les principaux travaux réalisés dans le sens de l'impact du capital humain sur la croissance économique, et l'incidence des facteurs reliés le capital humain à la croissance tel que les aspects qualitatifs du système éducatif et la nature de l'économie.

\section{A. Les différentes approches du capital humain}

Nous pouvons distinguer deux approches en termes d'éducation selon (Aghion et Howitt, 2000) :

- Première approche est fondée en flux du capital humain: Pour (Lucas, 1988), (Romer, 1989), (Barro ,1991), (Mankiw, Romer et Weil,1992), (Benhabib et Spiegel,1994), (Barro et Martin,1995) utilisent les variables de flux en se basant sur les valeurs du taux de scolarisation primaire, secondaire et supérieur, le taux d'alphabétisation, le ration d'encadrement, l'évolution de la démographie, le taux de mortalité compris entre 0 et 4 ans et enfin la fécondité.

- Deuxième approche est basée sur le stock du capital humain : À l'encontre de la première approche, cette deuxième approche a utilisée les variables de stock, à savoir que (Barro,1994), (Borenszensztein, De Gregorio et Lee,1994), (Bahalla,1995), (Lau, Jamison, Liu et Rivkin,1993), (Bloom et Mahal,1995) duquel le proxy recouru pour approcher le capital humain, c'est le pourcentage des individus en âge de travailler (scolarisés dans le secondaire).

Pour notre présente recherche, nous avons utilisé la première approche du capital humain du fait la disponibilité des données de ce sens.

\section{B. Considérations théoriques e la relation entre éducation et croissance économique}

Le capital humain est un bien immatériel qui peut faire progresser ou soutenir la productivité, l'innovation et l'employabilité. Il peut croître, se réduire ou devenir obsolète. Il provient de différentes origines, en l'occurrence et notamment, l'apprentissage organisé dans le cadre de l'éducation et de la formation.

Selon la théorie du capital humain (Becker, 1994) considère que l'éducation comme un investissement que l'individu doit acquérir d'une manière rationnelle pour se bâtir d'un capital productif inhérent de sa personne. L'étudiant est analogue à une firme intitulant un niveau initial en un stock de capital humain. C'est identique à une firme voudrait augmenter sa capacité productive qui peut se faire par l'investissement qui accroit son capital physique. En revanche, l'étudiant agit lui-même sur son stock de capital humain par le nombre d'années parcouru en éducation. Cette dernière renforce les aptitudes productives de la population active, en augmentant leur savoir-faire, leurs capacités et compétences et par conséquence se répercute sur le niveau de croissance.

La théorie d'accumulation du capital humain (Lucas, 1988) suggère que l'accumulation du capital humain et le savoir-faire sont des facteurs capitaux pour les niveaux de la productivité et de la croissance. Ils sont 
également considérés comme des facteurs de référence pour apprécier l'écart dans les taux de croissance et dans les niveaux de développement entre pays. L'instruction est considérée comme une bonne rivale et à prérogative d'usé1. L'éducation est donc un produit incorporé aux individus en tant que capital humain².

Selon le modèle de (Romer,1986) qui s'est basé sur l'éducation du niveau supérieur qu'est la source de la diffusion des connaissances et de l'innovation dans ses investigations en ignorant la décadence des rendements. À l'opposé du modèle de Lucas, la connaissance ou le savoir est connait un bien non concurrent, dont l'utilisation de la connaissance par un agent n'évite pas d'être utiliser par un autre. C'est aussi une propriété exclusivement partielle, du fait qu'il est possible d'entraver l'utilisation de la connaissance pour la production de biens, ceci est des fois difficile pour que cette connaissance soit utiliser pour produire une connaissance. Le droit de la propriété est partiel. En effet, la croissance est prévue comme le résultat principal de l'accumulation des connaissances. Un pays dédiant une place important de son capital humain à la recherche par le biais de l'enseignement supérieur provoquera une tendance à accroître plus vite qu'un autre. En fait, l'innovation et le progrès technique sont envisagés comme la conséquence des activités d'un secteur de formation de haut niveau et de la recherche qui vise à inventer et à produire de nouvelles «idées » ou de nouvelles « connaissances ».Ces dernières contribuant à l'accroissement du stock de capital humain national novateur capable d'améliorer et d'augmenter la productivité de l'économie nationale qu'est le conséquent de la disparité des niveaux de la croissance entre les pays.

Dans le modèle (Aghion et Howitte, 2000), qui adopte une idée qui étaye à Schumpeter dans leur modèle, en sorte que les innovations industrielles appuyant à l'amélioration de la qualité des biens produits sont des facteurs de croissance. L'idée fondamentale est que le progrès technique rend les biens produits antérieurement obsolètes. Selon Schumpeter, nous appelons aussi « l'approche fondée sur l'innovation » dans le cadre de cette approche, la croissance est reliée au stock du capital humain de haut niveau d'éducation qui résulte la capacité d'un pays d'innover et de se rattraper le retard à l'égard des pays développés et voir sa croissance grimper.

Les fondateurs du modèle d'ouverture commerciale (Berthélemy, Dessus et Varoudakis (1997) maintient deux principales idées. La première idée révoque le modèle de $\mathrm{MRW}^{3}$ pour étudier le lien entre l'accumulation du capital humain et la croissance. La deuxième dévoile le rôle indéniable du régime commercial. En fait, ils ajustent leur analyse dans un concept des échanges internationaux reposé sur l'introduction des variables interactives. C'est-à-dire que le niveau d'éducation élevé d'un pays en développement n'est pas suffisant pour atténuer l'écart avec les pays développés. Ceci réclame l'existence d'une économie ouverte pour stimuler l'impact du capital humain sur la croissance. Autrement-dit, le capital humain exerce son effet sur la croissance dans une économie fermée, mais contribue plus si la cadence d'ouverture commerciale est élevée.

Les fondements du modèle (Barro, 1990) invoquent le rôle de la qualité du système éducatif par le biais du volume des dépenses publiques consacrées à l'éducation, en particulier à travers l'investissement public en infrastructures, dans l'amélioration des acquis des individus, de la circulation de l'information, et dans l'accroissement des individus éduqués et à l'impulsion sociale. En effet, il renvoie un effet positif par le biais des indicateurs qualitatifs du capital humain admettent d'expliquer la différenciation de la croissance économique entre pays. L'attribut de l'éducation à la croissance et l'écart entre pays résulte à la fois quantitatif et qualitatif. Il divulgue que l'amélioration de la qualité du système éducatif entraine une influence positive sur la croissance économique on introduisait des variables qualitatives liées au système éducatif.

\footnotetext{
${ }^{1}$ Marielle Monteils, Loc. Cil., p. 2.

233 Ibid, p. 3

${ }^{3}$ Mankiw, Romer et Weil (1992).
} 


\section{Etudes empiriques sur les facteurs susceptibles d'influencer la croissance économique}

Knight, Loayza et Villanueva (1992), dont l'objectif de leur recherche est de tester la théorie néoclassique de la croissance économique par des données panel sur un échantillon de 98 pays entre 1960 et 1980, ainsi que le capital humain est rapproché par le pourcentage des personnes en âge de travailler (scolarisées dans le secondaire), ont trouvé des effets positifs de cette dernière sur la croissance d'ordre de $3.8 \%$. De même, (Benhabib et spiegel, 1994) ont rapproché le capital humain par le nombre moyen d'années d'études sur 78 pays incluant la période comprise entre 1965-1985 à travers une estimation de panel, ont trouvé l'effet significatif du capital humain à la croissance du revenu par tête qui résulte l'impact sur la croissance.

Selon (Than, 1995) en rapprochement du modèle Solow en-vue d'enlever les déterminantes majeures de la croissance en Malaisie entre la période 1971 et 1987 sur des données de panel. Il a montré que la croissance du capital physique à contribuer à la croissance d'ordre de 104\%. Or, (Oukaci, Bouznit et Abderrahmani, 2016) en Algérie en employant l'approche de Solow, ils montrent que la formation brute du capital fixe est l'unique coefficient de ce derrière en corrélation avec le produit intérieur brut qui vaut un taux de $90 \%$.

Selon (Vandenbussche, Aghion et Meghir, 2006), qu'ont mis l'accent de la place de l'enseignement supérieur dans les activités d'innovation et d'imitation dans leur modèle. En fait, ils ont montré que l'utilité et le développement de l'enseignement supérieur ont devenu fondamentaux pour chaque pays lorsqu'il est éloigné de la frontière technologique, car l'imitation commanderait l'innovation pour joindre la frontière technologique. Autrement-dit, quand le pays se rejoint de la frontière technologique est que l'éducation au niveau des universités précipite l'adoption de nouvelles technologies ce qui contribue à la croissance. De ce fait, plus le nombre d'effectifs des étudiants est grand plus le pays se lance à l'innovation qui résulte à l'amélioration de la balance commerciale et par conséquent booste la croissance. À l'opposé, (Boulia et Haddar, 2006) qui ont mené une étude sur le lien entre l'enseignement supérieur et la croissance économique par l'usage tant indicateurs de l'enseignement supérieur le taux d'effectifs étudiants et les dépenses consacrées en éducation supérieure, et la croissance économique est rapprochée par le PIB réel par tête. Ils ont parachevé l'inexistence de relation entre l'éducation et la croissance économique dont l'éducation est loin de se manifesterait en tant qu'un facteur de croissance à court terme et à long terme dans le système éducation tunisien.

Pour (Abdouni et Hanchane, 2006) ont introduit dans leur équation de croissance la variable qui représente l'ouverture commerciale internationale à travers l'addition les exportations avec les importations du fait qu'ils ont accentué sur l'effet non significatif du capital humain sur la croissance économique. Les estimations économétriques attachées au coefficient de cette variable est toujours positif et significatif ce qui permet de dire que plus le taux d'ouverture commerciale est élevé des pays en développement est plus l'accès au savoir et la rapidité de convergence seront précipités et susceptibles d'impacter le taux de croissance.

Les indicateurs de la qualité des systèmes éducatifs comme souligne le rapport «Education Report de l'Unesco, 1997 » appuie sur deux parmi d'autres qui se présentent en termes monétaires en concrétisant l'effet de la nation en matière d'éducation. Il s'agit notamment des dépenses publiques d'éducation en pourcentage des dépenses du gouvernement et les mêmes dépenses en pourcentage du PIB. Ainsi que parmi les indicateurs, il existe deux autres qui sont le taux de croissance de la population dans les tranches d'âge entre (0-14) et de (plus de 65 ans) qui permettent de donner un aperçu de la relation offre et demande d'éducation d'un pays et un dernier qui est le taux d'encadrement qui permet de diagnostiquer les conditions d'enseignement d'un pays selon l'hypothèse sous-jacente que les meilleurs acquis s'effectués dans les établissements et classes dont le nombre d'élèves ou étudiants est réduit. 
Par l'approche de Slow (Tham Tham, 1995) à essayer d'analyser les déterminants susceptibles qui influencent la croissance en Malaisie pendant la période entre 1971 et 1987. Les estimations économétriques de son étude dévoilent au-delà des variables de travail et de capital, les dépenses publiques consacrées à l'éducation a contribué à la croissance à $18 \%$. Cette variable paraît donc comme le véridique mécanisme de la croissance Malaisienne sur la durée de l'étude. L'augmentation de la productivité du facteur travail quant à elle est demeurée négliger et ceci à cause de l'utilité proactive de dépense publique. Pour leur part (Meghir,2007) a dévoilé le rôle majeur des dépenses publiques consacrées à l'éducation supérieure du fait qu'une augmentation de 0.7 point du PIB des dépenses dans l'éducation engendre une croissance en passante de $1.3 \%$ à $2 \%$ du PIB.

Pour (Blanchet, 1988) dans ces travaux à également focaliser sur l'appréciation de l'influence de la croissance démographique sur la croissance économique. Son étude est menée sur 78 pays en voie de développement couvant la période entre 1960 et 1980 à travers laquelle a trouvé l'influence de la croissance de la population dont le taux de croissance de cette dernière est décalé de 10 ans et 20 ans sont strictement corrélés, à l'encontre de celle de plus de 60 ans. Les premiers ont des impacts positifs sur la croissance pendant toute la période sauf la période 1970-1980.

\section{Hypothèses à vérifier de l'étude :}

À partir du développement de la revenue littérature, nous supposons que la convergence quantitative et qualitative de l'éducation en particulier au niveau supérieur tant que l'ouverture des économies possibles d'affecter la croissance économique. Or, nous pouvons établir une série d'hypothèses que nous allons chercher à tester dans le cadre de cette recherche.

- H1 : Le stock du capital humain en particulier du niveau supérieur influencerait positivement la croissance économique.

- H2 : L’investissement en capital physique impacterait positivement la croissance économique.

- H3 : Plus l'ouverture commerciale est élevée, plus la croissance économique serait robuste.

- H4 : Plus les dépenses publiques déduites à l'enseignement supérieur sont importantes, plus la croissance économique serait éminente.

- H5 : Un encadrement atténue affecterait positivement la croissance économique

- H6 : Plus le taux de croissance de la population en âge de scolarisation supérieure est élevé, plus la croissance économique serait performante.

\section{PRESENTATION DES DONNEES ET CADRE DE MODELISATION}

Dans le cadre de cette partie, nous donnons un aperçu sur la source de nos données usées à travers laquelle nous avons effectué les différents traitements économétriques ${ }^{4}$, la méthodologie adoptée à notre thématique, ainsi que le modèle d'estimation de la croissance.

\section{A. Présentation des données}

Pour collecter les informations nécessaires pour notre présente étude empirique portée sur quatre pays d'Afrique qui sont : le Maroc, la Tunisie, le Sénégal et la Niger, nous Somme basé sur la base de données qui provienne de la banque mondiale couvrant la période (2002-2018). Dont les variables retenues sont exclusivement choisies en fonction de la revue littérature citée auparavant à savoir :

${ }^{4}$ L'ensemble des régressions ont été effectuées à l'aide du logiciel Stata 
- Variable endogène:

La croissance mesurée par le PIB/tête.

- Variables exogènes

Capital humain: est représenté par le stock de capital humain disponible qui est apprécié à travers le taux de scolarisation (nombre d'étudiants)

Le capital physique : est estimé à travers la formation brute fixe (FBCF) qui est le taux d'investissement en capital physique

L'ouverture commerciale : est évaluée par l'addition des exportations et des importations en pourcentages du PIB

Les dépenses publiques : il concerne les dépenses consacrées à l'éducation supérieure du total de pourcentage des dépenses gouvernemental

Le taux d'encadrement : est représenté par le ration étudiants/professeurs de l'enseignement supérieur

Le taux de croissance de la population : les pourcentages de la population âgée de plus de 65 ans et entre 0 et 14 ans dans la population totale influenceraient la croissance.

B. Présentation de la méthode d'estimation des données de panel

D’une façon générale, le modèle économétrique linéaire des données de panel est défini comme suit :

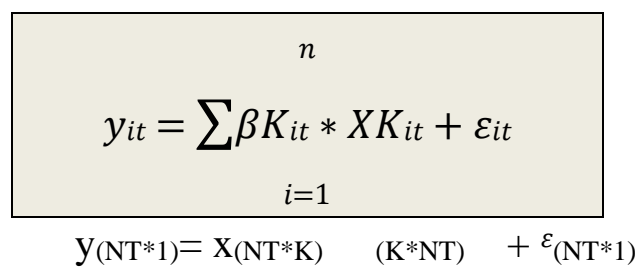

$y_{i t}$ : représente la variable dépendante pour l'individu i à la période $\mathrm{t}$

$X K_{i t}$ : indique la $\mathrm{k}^{\text {ième }}$ variable explicative pour l'individu i à la période $\mathrm{t}$

$\mathbf{I}=1,2,3, \ldots, \mathrm{N}$ (N le nombre d'individus)

$\mathbf{T}=1,2,3, \ldots, \mathrm{T}$ ( $\mathrm{T}$ le nombre de périodes $)$

Ainsi que pour une estimation économétrique convergente et bonne sur des données de panel, nous serons menés à passer par deux étapes :

En première étape : nous vérifions s'il s'agit bien d'un modèle panel à effets individuels ou d'un modèle Pooling par la méthode du multiplicateur de Lagrange dont l'application au problème là a été induite au teste (Breusch et Pagan, 1979) sur logiciel STATA qui vaut une probabilité statistique au seuil de $5 \%$ pour l'acceptation d'une structure à erreurs composées. Dans notre analyse, nous avons dégagé une valeur de la probabilité du teste Breush-Pagan de 0.00 qu'est inférieure à $5 \%$ (voir l'annexe 1) donc il accepte la structure à erreurs composées. À cet égard, nous passons à la deuxième étape.

En deuxième étape : il s'agit à choisir entre le modèle à effets fixes et le modèle à effets aléatoires avec lequel qui sera le plus approprié à nos données de panel. Pour ce faire, nous recourons au teste de spécification 
d'Hausman dont les coefficients deux estimations à savoir fixe et aléatoire sont statistiquement distincts. L'idée sous-jacente de ce teste est que, l'hypothèse nulle d'indépendance ente les erreurs et les variables exogènes, si la probabilité de la statistique est supérieure au seuil de confiance qu'est $5 \%$, les deux estimations sont non biaisés, en conséquence les coefficients estimés devraient peu différer ce qui résulte à des effets aléatoires dont le modèle opérant est le modèle Moindre carres Généralisés (MCG).Et si l'inverse, il s'agit des effets fixes dont le modèle opérant, c'est le modèle White. De ce fait, notre analyse se manifeste par un modèle à effets fixes qui est plus approprié à nos données puisque la $\mathrm{P}$-value est inférieure au seuil de $5 \%$ (voir annexe 2). Il est mieux donc de tenir les estimations du modèle à effets fixes (sans biais) car ceux du modèle à erreurs composées sont biaisés.

\section{Spécification du modèle}

Nous recourons au modèle néoclassique de croissance développé par (Islam, 1995) qui nous permettrons de combiner à la fois des effets individuels et des effets temporels dont en tirant davantage de l'analyse en panel. Il est particulièrement une spécification du modèle de (Mankiw et al, 1992) pourtant sur des donnés de panel. Ces derniers à leur tour ont pris les soubassements du modèle de (Solow, 1956), dans lequel ils insèrent la notion du capital humain. A cet égard, deux types de capital sont cependant incorporés. Il s'agit du capital physique et du capital humain, dont la fonction de production est de type Cobb-Douglas :

$$
\begin{gathered}
\mathrm{Y}=\mathrm{A} \mathrm{K}^{\mathrm{a}} \mathrm{L}^{\mathrm{b}}(1) \\
\mathbf{Y}: \text { Croissance } \\
\mathbf{K}: \text { Capital physique } \\
\mathbf{L}: \text { Capital humain }
\end{gathered}
$$

En conséquence, la spécification de la fonction de production de Cobb-Douglas, avec lequel la production ou la productivité en logarithme se décrit linéairement en fonction des intensités des facteurs, duquel la spécification de la relation en logarithmique (1) est la suivante :

$$
\log =\log \mathrm{A}+\mathrm{a} \log \left(\mathrm{k}_{\mathrm{t}}\right)+\mathrm{b} \log \left(\mathrm{L}_{\mathrm{t}}\right)+\text { ex...(2) }
$$

Après avoir présenté les différentes variables retenues dans notre présent travail, nous posons la fonction suivante :

$$
\begin{aligned}
& \ln (\text { GDPHt })=\mathbf{a} 0+\mathbf{a} 1 \ln (\text { FBCFt })+a 2 \ln (\text { TSPNt })+a 3 \ln (\text { TSSt })+a 4 \ln (\text { TSUt })+a 5 \ln (\text { DPEt })+a 6 \\
& \ln (\mathrm{TXE})+\mathbf{a} 7 \ln (\mathrm{TCPt}) \mathrm{a} 8 \ln (\mathrm{CEXGDPt})+\mathrm{a} 8 \ln (\mathrm{TCP} t)^{2}+\mathrm{a} 9 \ln (\mathrm{TCH})+\mu \mathrm{t} . \mathrm{Où} \mathrm{:}
\end{aligned}
$$

$\mathrm{T}: 2002 ; \ldots \ldots ; 2018 \mathrm{U}_{\mathrm{t}}$ : c'est le terme d'erreur du modèle

$\mathbf{a}_{1} ; \mathbf{a}_{2} ; \ldots \ldots . . . \mathbf{a}_{5}$ : sont les coefficients qui interprètent les impacts de variation des variables explicatives sur la croissance.

- $\ln (\mathrm{GDPH})$ indique le logarithme du PIB réel par habitant de l'année $t$

- $\log (\mathrm{FBCF})$ indique le logarithme de la formation brute du capital fixe à l'année $\mathrm{t}$

- $\ln (\mathrm{TSU} t)$ indique le logarithme du taux de scolarisation universitaire de l'année t

- $\ln (\mathrm{DPEt})$ indique le logarithme de la dépense publique consacrée à l'éducation supérieure de l'année

- $\quad \ln (\mathrm{TXE})$ indique le logarithme du taux d'encadrement (professeurs/étudiants)

- $\ln (\mathrm{TCP} t)$ indique le logarithme de la part de la population de l'année t âgée plus de 65 ans

- $\quad \ln (\mathbf{T C P})^{2}$ indique le logarithme de la part de la population de l'année t âgée moins de 14 ans

- $\ln (\mathrm{CEXGDPt})$ indique le logarithme du taux d'ouverture (exportation+importation/PIB) de l'année $\mathrm{t}$

4. RÉSULTATS ET INTERPRÉTATIONS

Nous présentons les résultats des estimations effectuées ainsi que les interprétations des résultats relatives à la croissance économique. 
ELKHALFI et al.: L'impact de l'Enseignement Supérieur sur la Croissance Economique...

\section{A. Présentation de l'effet d'enseignement supérieur sur la croissance économique}

Nous regroupons les trois estimations économétriques dégagées de l'effet de l'enseignement supérieur sur la croissance économique ci-après.

TAB.1. ESTIMATION DE L'EFFET DE L'ENSEIGNEMENT SUPERIEUR SUR LA CROISSANCE ECONOMIQUE

\begin{tabular}{|c|c|c|c|c|c|c|}
\hline \multirow{2}{*}{$\begin{array}{l}\text { Variables } \\
\text { Explicatives }\end{array}$} & \multicolumn{2}{|c|}{ Modèle à effets fixes } & \multicolumn{2}{|c|}{ Modèle à effets aléatoires } & \multicolumn{2}{|c|}{ White (avec correction) } \\
\hline & Coefficients & Std. Err. & Coefficients & Std. Err. & Coefficients & Std. Err. \\
\hline Taux de scolarisation supérieur & $.0185526^{* * * *}$ & .057429 & $.003456^{*}$ & .0080794 & $.0185526^{* * * *}$ & .057429 \\
\hline Taux d'investissement & $.00525604^{\mathrm{NS}}$ & .0146369 & $.025348^{* * * *}$ & .004467 & $.0229004^{*}$ & .0236369 \\
\hline Ouverture commerciale & $.0069432^{* * *}$ & .028895 & $.0219098^{* * * *}$ & .038126 & $.0069432^{* *}$ & .028895 \\
\hline Dépenses publiques consacrées ES & $.005481^{* *}$ & .023241 & $.0109236^{* * * *}$ & .039346 & $.005481^{* * *}$ & .022912 \\
\hline Taux d'encadrement & $-.0053427^{* *}$ & .022912 & $.001891 *$ & .0039403 & $-.0053427^{* *}$ & .023241 \\
\hline Démographie âgée plus de 65 & $-.066792^{* * *}$ & .019651 & $.0019343^{\mathrm{NS}}$ & .0033248 & $-.066792^{* * *}$ & .019651 \\
\hline Démographie âgée moins de14 & $.0159478^{\mathrm{NS}}$ & .0170605 & $.0177579^{\mathrm{NS}}$ & .0281915 & $.0179478^{*}$ & .0195060 \\
\hline Constante & $6.166904^{* * *}$ & .1960939 & $5.179158^{* * * *}$ & .1930992 & $6.166904^{* * *}$ & .1960939 \\
\hline $\mathbf{R}^{2}$ & & 0.6841 & & 0.9572 & & 0.6841 \\
\hline Fisher & & alue : 0.000$)$ & & lue : 0.000$)$ & & lue : 0.000$)$ \\
\hline
\end{tabular}

Source : établie à partir de nos données ${ }^{* * *}$ : significatif à $1 \%,{ }^{* *}:$ significatif à $5 \%,{ }^{*}$ : significatif à $10 \%,{ }^{\text {NS}}$ : Non significatif.

\section{B. Interprétation des résultats}

Le modèle de Solow comme indique le tableau au-dessus est mieux spécifique avec le modèle à effets fixes qu'est plus approprié à la structure de nos données de l'échantillon suivant le teste de Hausman avec (R2 $=68.41 \%$ ), ce qui traduit que la variance de la croissance par tête est expliquée auprès de $68.41 \%$ par les variables explicatives du modèle. Le teste Fisher est significatif avec une (P-value : 0.0000) ce qui prouve que le choix des variables est pertinent pour expliquer la croissance par tête. Le teste de student nous montre que notre modèle contient deux variables significatives corrigées de l'hétéroscédasticité par la méthode de White ce qui confirme la performance de cette dernière.

Les résultats de la deuxième estimation avec la méthode White montrent que la majorité de facteurs ont gardé le même effet par rapport à la première estimation à effets fixes. IL s'agit des variables « Taux de 
scolarisation supérieur», «Ouverture commerciale», «Dépenses publiques consacrées à l'enseignement supérieur », «Taux d'encadrement» et «Démographie âgée plus de 65 ». Sauf pour les variables du taux d'investissement en capital physique et population âgée mois de 14 ans qui sont devenus significatives avec correction. Comme est déjà signalé dans le paragraphe de la méthode d'estimation que nos données s'opéreraient à effets fixes d'où que notre analyse porte sur la variabilité de la croissance du modèle white avec correction (effets fixes).

- En effet, la variable « Taux de scolarisation supérieur », a un impact positif sur la croissance par tête avec un coefficient significatif au seuil de $1 \%$, ce qui ce qui rejoint l'hypothèse 1 , ainsi que les modèles théoriques de croissance endogènes (Romer, Lucas et Aghion, 1986, 1988, 2000) et les travaux empiriques de (Benhabib et Spiegel, 1994), (Knight, Loayza et Villanueva, 1992) et (Vandenbussche, Aghion et Meghir, 2006) qui démontrent une parfaite concordance entre le capital humain supérieur et la croissance par tête, du fait que plus les individus passeraient par l'enseignement supérieur plus ils auraient un stock de capital humain disponible basé sur l'innovation et le progrès technologique, ce qui affecte d'une part ,la productivité de l'économie nationale et de se rattraper par rapport aux autres pays développés en améliorant la qualité de la main d'œuvre et par conséquence la croissance par tête. En d'autre part, il affecte la composition des compétences de haut niveau que pouvant tirer profit des retombées positives des flux d'IDE susceptibles d'influencer le niveau du PIB par tète. Et contredire les aboutissements de ((Boulia et Haddar, 2006) qui stipulent l'inexistence de relation entre l'éducation supérieure et la croissance économique. D'une manière globale, le passage d'une grande part de la population par l'enseignement supérieur offre un niveau d'éducation élevé capable à inciter les pays en voie de développement à adapter des stratégies d'imitations en exploitant des technologies existantes par le biais des formations professionnelles, techniques et d'une particularité sectorielle visant à réduire le chômage et à favoriser l'innovation, l'investigation et le développement dans divers domaines, notamment de santé, de l'industrie, d'exportation, et d'information, etc, ce qui attribue à la croissance économique.

- Pour la variable «Taux d'investissement en capital physique», nous constatons qu'elle a un coefficient significatif au seuil de $10 \%(\mathrm{t}=2.36)$ par rapport à la première régression qui se manifeste non significatif ( $\mathrm{t}=1.46)$. Ce résultat est cohérent avec l'hypothèse 2 et les études empiriques de (Tham ,1995) et (OUKACI, BOUZNIT et ABDERRAHMANI ,2016) qui stipulent que l'élargissement de l'appareil productif conditionne la productivité et par conséquence la croissance. En effet, un capital de compétences de haut niveau d'éducation permet d'adopter deux politiques, soit la politique d'imitation en adaptant des technologies déjà existante, soit politique d'innovation en procréant de nouvelles technologies ce qui retombe l'augmentation de la capacité physique qui favorise à sont tour la capacité de productivité innovante ce qui contribue à la croissance. Économiquement, c'est la complémentarité des facteurs de production qui sont (travail, capital).

- Le signe positif et significatif $(\mathrm{t}=3.81)$ du coefficient de la variable « Ouverture commerciale » exprime une relation linéaire et positive entre le degré d'ouverture commerciale des pays et la croissance par tête. Ce constat nous met en accord avec l'hypothèse 3 et le modèle de (Berthélemy, Dessus et Varoudakisre, 1997) qui suggèrent que le stock de compétences figure l'influence sur la croissance si le degré d'ouverture commerciale est élevé. Ces résultats aboutissent au même sens que les travaux de (Abdouni et Hanchane, 2006) qu'ont dévoilé les retombées positives de l'ouverture économiquement des pays qui se manifeste par une rapidité pour l'accès au savoir et pour la vitesse de convergence ce qui contribue à la croissance. Dans cette optique, nous pouvons 
dire que les externalités du capital humain véhiculent aussi bien par l'ouverture commerciale que par les IDE seront également mieux exploiter, ce qui permet de bien tirer davantage de cette composante au profit de son stock du capital humain sur la croissance.

Concernant la qualité des systèmes de l'enseignement supérieur, cette qualité est présentée à partir de quatre indices cités dans le rapport de l'UNISCO (1997). Dont deux de ces indicateurs évoquent l'effort de la nation en éducation, en termes monétaires. Il s'agit des dépenses publiques d'éducation en pourcentage du PIB ou les mêmes dépenses en pourcentage des dépenses du gouvernement et l'encadrement. Pour notre investigation, nous avons retenu les dépenses publiques d'éducation en pourcentages des dépenses gouvernementales dont la variable que nous disposant dans notre base de données. Et, dont deux reflètent l'état démographique des pays. Il s'agit de la population âgée de 0 à 14 ans; et la part de la population âgée plus de 65 ans, dans la totalité de la population. Ces deux indices ont été élaborés afin de mettre en relation offre et demande d'éducation.

- Nous commençons par la première variable de critère de qualité qu'est les « dépensées consacrée à l'enseignement supérieur », nous constatons qu'elle a un effet positif et significatif ( $\mathrm{t}=2.29)$ sur la croissance par tête. Ce résultat confirme le modèle de (Barro, 1990) qui stipule que l'augmentation de la qualité de l'enseignement à travers l'investissement public en infrastructures en matière d'équipements et dans la recherche entraîne une augmentation de la croissance. Ce qui aboutit dans le même sens que la notre 4ème hypothèse. Ce qui mit l'accent sur l'implication du rôle de capital humain qualité tant que quantité aura des effets avantageux sur la croissance, et avec un impact assez fort pour le capital physique vu que la manipulation de ce dernier est sophistiquée pour tous les pays sélectionnés. Ce constant nous met aussi en accord avec les études effectuées par (Tham Tham, 1995) et (Meghir ,2007) qui justifient l'effet positif des dépenses dans l'éducation supérieure sur la croissance.

- IL faut noter que le variable «taux d'encadrement» semble exister, en effet le signe négatif et significatif $(\mathrm{t}=2.32)$ du coefficient, ce dernier influence négativement sur la croissance par tête : Lorsque le taux d'encadrement augmente, le cadre enseignant prend en charge plus d'étudiants. En effet, cette augmentation engendre plus de responsabilités et plus de charges au corps enseignant ce qui peut se répercuter négativement sur l'encadrement et de là détériore la qualité de la formation acquise qui baisse la qualité des compétences disponibles pour attirer les IDE et qui bouscule la maitrise des secteurs innovants qui étalent des postes plus importants procurant une rémunération plus conséquente et par conséquent affecte négativement la croissance par tête. Ce constat correspond parfaitement aux résultats de nos estimations puisqu'une augmentation du taux d'encadrement de $1 \%$ conduirait à une baisse de la croissance part tête $(-0.5 \%)$. Ce dernier nous met en accord avec l'hypothèse 5 .

- Nous passons par variable démographique qui engobe de notre échantillon deux catégories de population à savoir : «population à mois de 14 ans » et « la population à plus de 65 ans ». Nous enregistrons, pour la première tranche de la population un effet négatif sur la croissance et pour la deuxième classe un effet positif largement minoritaire sur la croissance ce qui repris notre 6ème hypothèse. En effet, plus la part de ces deux catégories dans la population totale est grande, disposeraient-t-elles moins des individus passeraient du temps à la formation supérieure, moins ils auraient un stock de capital humain susceptible d'impacter le niveau du PIB/tête. Ces résultats sont approuvés par l'étude de (Blanchet, 1988) qui montre l'impact positif du taux de croissance de la population en tranche d'âge de scolarisation dans le supérieur sur le Pib par tête. 


\section{CONCLUSION}

Notre investigation de l'évaluation de l'enseignement supérieur dans une vision macro-économique et son effet sur la croissance, nous a poussé à deviner que, la qualité des systèmes éducations supérieures jouent amplement sur les rendements de la formation acquise et les trajectoires de croissance des pays en voie de développement. En effet, les résultats obtenus sur les quatre pays de notre échantillon à dévoiler que la qualité du système enseignement supérieur conduit largement à déterminer la forme des rendements.

Après la correction de notre estimation par le modèle white apparaît convenablement assuré les hypothèses de base pour la relation enseignement supérieur-croissance pour :

En premier, les résultats confirment largement l'effet du nombre d'étudiants dans l'enseignement supérieur sur le l'ampleur de la productivité et ca nous a mené que les années d'études effectuées au niveau de l'enseignement supérieur sont les motrices vitaux de la croissance économique.

En deuxième, l'ouverture des économies est admise tant qu'une variable significative avec un signe positif. Le capital humain du niveau supérieur s'améliore son effet sur la croissance si le degré d'ouverture commerciale est élevé.

En troisième, le modèle à démontrer l'influence négatif de la population hors d'âge de scolarisation supérieure sur la croissance économique.

En dernier, l'estimation confirme que l'effet de l'enseignement supérieur sur la croissance et s'amélioration de la relation éducation-croissance est indexé au premier lieu à la qualité du système éducatif supérieur de ces pays. Notamment, les efforts de ces pays en termes monétaires et qualité de la formation qui améliore la contribution de la formation supérieure sur le Pib par tête. Ce qui nous prouve que l'enseignement supérieur comme pont entre l'investigation, l'innovation et la créativité, qui admettrons de rattraper les pays développés et de demeurer dans la troupe de tête, ainsi que pour faire face aux effets de concurrence et de compétitivité.

La limite de notre recherche réside du fait de la restriction de notre échantillon, qui pourra être ultérieurement menée dans des investigations en matière dans des échantillons vastes.

\section{ANNEXES}

Annexe 1 : le teste de BREUSCH et PAGAN pour le modèle

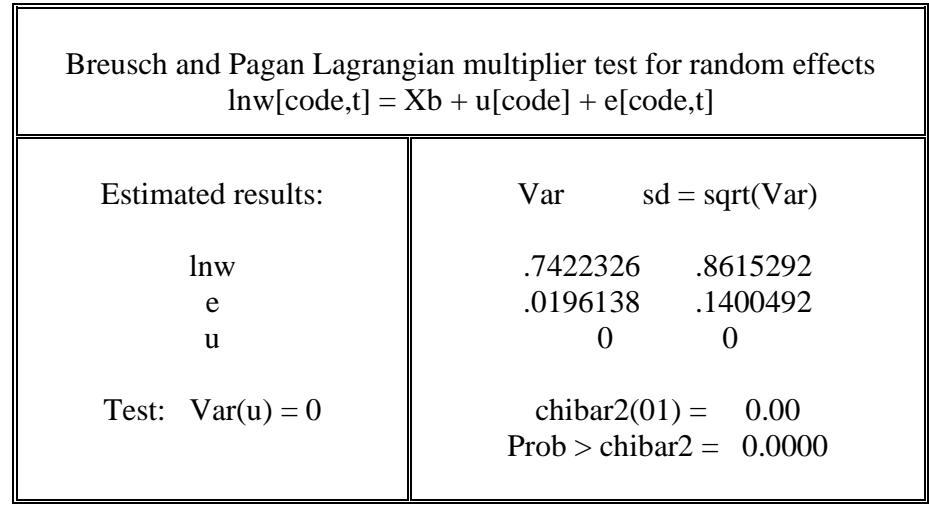

Source : élaboré à partir de nos données 


\section{Annexe 2 : le test de Hausman}

\begin{tabular}{|c|c|c|c|c|}
\hline & \multicolumn{4}{|c|}{----- Coefficients ---- } \\
\hline & \multicolumn{4}{|c|}{ V_B) } \\
\hline & re & & Difference & S.E. \\
\hline Tauxdescol r & .003456 & .0185526 & - -.0150966 & (.0056829 \\
\hline FBCF & .0253489 & .0225604 & .0027885 & $=$ \\
\hline OC & .0219098 & .0069432 & .0149666 & .0024873 \\
\hline REES & .0018912 & -.0053427 & .0072339 & .0031748 \\
\hline DCEE & .0109236 & .005481 & .0054427 & .0032056 \\
\hline Dmographie1 & .0019343 & 0066792 & -.0047449 & .0027524 \\
\hline Dmographie3 & .0177579 & -.0169478 & .0347056 & .0224433 \\
\hline \multicolumn{5}{|c|}{ 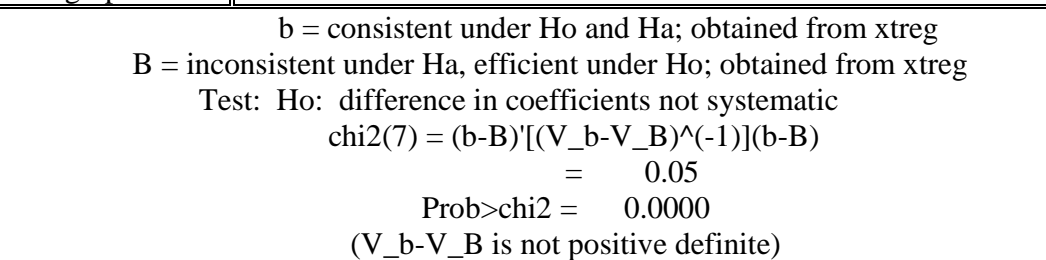 } \\
\hline
\end{tabular}

\section{Bibliographie}

[1] Abdouni et Hanchane (2006) « Ouverture, capital humain et croissance économique »: fondements théoriques et identification des liens à l'aide de données de panel

[2] Aghion P et Howitt P. (2000), Théorie de la croissance endogène, éditions Paris.

[3] Aghion, P et Cohen, E (2004), Education et Croissance, Conseil d'Analyse Economique

[4] Barro, R. (1991), Economic Growth in a Cross Section of Countries, Quarterly Journal of Economics, 106, pp. 407-443.

[5] Barro, R.J et Sala-i-Martin, Xavier (1994), Quality Improvements in Models of Growth, NBER Working papers $n^{\circ} 4610$.

[6] Becker G.S (1964) Investment in human capital: A Theoretical Analysis, Journal of Political Economy 70 pp, 9-49.

[7] Benhabib J. \& Spiegel M.M (1994) The Role of Human Capital in Economic Development: Evidence from Aggregate CrossCountries Data, Journal of Monetary Economics, vol 34, n², pp. 143-173.

[8] Berthélemy JC., Dessus S. et Varoudakis A.(2007) Capital humain, Ouverture Extérieure et Croissance : Estimation sur données de panel d'un modèle à coefficients variables, revue économique; vol. 48, No 3.Centre de Développement de l'OCDE pp 419-428.

[9] Boulila Ghazi et Haddar Mohamed (2006), Enseignement Supérieur et Croissance Economique : Le Cas Tunisien, Nations Unies Commission Economique Pour L'Afrique : IDEP-DAKAR.

[10] Hans-Jürgen Engelbrecht, (May 2001), "The l'ole of human capital in economic growth: Some empirical evidence on the "Lucas vs. Nelson-Phelps" Controversy", Massey University, Department of Applied andInternational Economies, Discussion Paper No. 01. 02, p. 2.

[11] Jean Luc de Meulemeester et Claude Diebolt, (2007), «Éducation et croissance: Quel lien, pour quelle politique? », Associationfrançaise de Cliométrie, Working papers $n^{\circ} 8$, p. 3.

[12] Jean-Claude.B, Sébastien et Aristomène.V, (1979) CAPITAL HUMAIN, OUVERTURE EXTÉRIEURE ET CROISSANCE : estimation sur données de panel d'un modèle à coefficients variables, Editions de l'OCDE

[13] Lucas R.E. (1988) On the Mechanics of Economic Development, Journal of Monetary Economics, $\mathrm{n}^{\circ} 22$ (1), pp. 3-42. 
[14] Mankiw, N.G., Romer, D., Weil, D.N. (1992) A Contribution to the Empirics of Economic Growth, Quarterly Journal of Economics, ${ }^{\circ} 107$ (2), pp. 407-437.

[15] OUKACI, BOUZNIT et ABDERRAHMANI en Algérie (2016les cahiers du cread $\mathrm{N}^{\circ} 113 / 114$ )

[16] Romer, P. (1990), Endogenous Technological Change, Journal of Political Economy, vol.98, Supplement, p. 71-102.

[17] Schultz, T., 1961, Investment in Human Capital, American Economic Review, 51, 1-17.

[18] Solow R. (1956), A Contribution to the Theory of Economic Growth, Quarterly Journal of Economics, $\mathrm{n}^{\circ}$ 70, pp. 65-94.

[19] Sylvie CHARLOT(1997), « La relation éducation-croissance:apports théoriques récents et tests empiriques » UNIVERSITE DE BOURGOGNE.

[20] Vandenbussche, J.Aghion, P, Meghir,C. (2006), Growth, distance to frontier and Composition of Human Capital. Journal of Economic Growth, 11, 97-127.

[21] Weil David (2005), Accounting for the Effect of Health on Economic Growth, NBER Working papers 11455 (National Bureau of Economic Research 